\title{
Musculoskeletal Disorders of the Cervical Spine Following Neck Dissections Due to Malignancies of the Head And Neck
}

\author{
Zornitsa Mihaylova \\ ${ }^{1}$ Department of Dental, Oral and Maxillofacial Surgery, Faculty of Dental Medicine, Medical University \\ - Sofia, Bulgaria
}

\begin{abstract}
:
Neck dissections are surgical interventions aiming the removal of lymph nodes from specific areas of the neck, together with non-lymphatic structures falling within the scope of the operation $-\mathrm{m}$. sternocleidomasstoideus (MSCM), v. jugularis interna (VJI), $\mathrm{n}$. accessorius (N.XI). Due to the involvement of important anatomical structures in the neck dissections, postoperative outcoomes and complications associated with impaired function of the musculoskeletal system of the neck arise.

The purpose of the present study is to determine the degree of cervical spine movements depending on the volume of surgical neck dissection.

Methods. The goniometry method was used to evaluate the motor function of the operated patients. The results obtained were analyzed via SPSS Vers23.0.

Results. A statistically significant difference was observed postoperatively at the $3 \mathrm{rd}$ month in all directions of movement studied in the three groups of operative interventions (neck dissections): flexion $\mathrm{F}(2,65)=643.566, \mathrm{p}<0.001$; extension $-\mathrm{F}(2,65)=445.045$, $\mathrm{p}<0.001$; lateral flexion $-\mathrm{F}(2,65)=314.077$, $\mathrm{p}<0.001$; rotation $-\mathrm{F}(2,65)=121.980, \mathrm{p}<0.001$.

Conclusion. Assessment of the cervical spine shows that all directions of movement are affected, most significantly affecting lateral flexion in all groups of operated patients with neck dissection.
\end{abstract}

Key words: active range of motion (AROM), neck dissection, cervical spine restricted motion, postoperative consequences

\section{Introduction.}

Neck dissections are recommended as surgical procedures for the removal of regional lymph nodes in the cervical region due to malignancies in the second half of the 19th century. In 1888 Franciszek Jawdynski described in detail an-bloc resection of the cervical metastatic mass [1,2]. In 1905, George Crile reported the treatment of 105 patients with head and neck cancers and 121 conducted radical neck dissections [3]. In 1952, Osvaldo Suarez proposed modified techniques for the preservation of non-lymphatic structures. He called them "functional" or "conservative" surgery [4, 5].

Neck dissections are surgical interventions for the removal of lymph nodes from specific areas of the neck, as well as the removal at a defined volume of operative intervention of non-lymphatic structures $-\mathrm{m}$. sternocleidomasstoideus (MSCM), v. jugularis interna (VJI), n. accessorius (N.XI). Due to the involvement of important anatomical structures in the neck dissections, undesirable outcomes and complications arise [6, $7,8,9]$. Assessment and analysis of adverse treatment outcomes are defined primarily as complications and target specific structures - nerves $[10,11]$ and blood vessels $[12,13,14]$.

Postoperative morbidity in the mobility of the cervical spine follows the occurrence of postoperative cicatrix, with damage to $\mathrm{m}$. sternocleidomastoideus (MSCM) and affecting n. accessories [15]. Due to the elasticity of the soft tissues, Wilgen et al. suggested little influence of the Active Range of Motion (AROM) cicatrixes on the neck region [14]. Patients with MSCM saccharification find non-significant reduction of AROM in the contralateral axial rotation [14]. 
The aim of this study is to determine the level of postoperative morbidity in the mobility of the cervical spine depending on the volume (type) of the neck dissection in patients with malignancies in the maxillofacial and cervical areas.

\section{Materials and methods.}

Patients. Patients with neck dissection due to metastases arising from malignant tumors in the maxillofacial and cervical areas were examined. The study includes the data for the period March 2016 - March 2019 at the Alexandrovska Hospital, Sofia. The following were selected as exclusion criteria in the selection of patients: previous radiotherapy, pre-operatively diagnosed disorders of the movement of the cervical spine, accompanying diseases of the musculoskeletal system. All patients completedand signed informed consent to participate in this study.

Methods. In the study of cervical spine motility, goniometry was applied to evaluate the musculoskeletal system - a method measuring angles when two or more bones move relatively to each other, by jointing them together [16]. Range of Motion (ROM) measurement of cervical spine was conducted, and Active Range of Motion (AROM) - an arc of motion achieved by unassisted articulation, was evaluated..

Instruments called goniometers are used to measure the degree of mobility between defined reference points. The following instruments were used for the purposes of this study:

- Universal goniometer, manufactured by Patterson Medical, consisting of two polymer plates, total length $32 \mathrm{~cm}$ (Fig. 1). The goniometer body has a round shape. The center scale is graduated at 1 degree intervals and measures from 0 to $180^{\circ}$ and from 180 to $0^{\circ}$. The outer scale (decorated in red) measures from 90 to $0^{\circ}$ and from 0 to $90^{\circ}$. The inner scale (decorated in red) measures 360 to $180^{\circ}$ and 180 to $360^{\circ}$. The stationary (main) arm of the goniometer is fixed to the body. The movable arm is connected through the joint at the center of the body and performs a free movement of $360^{\circ}$.

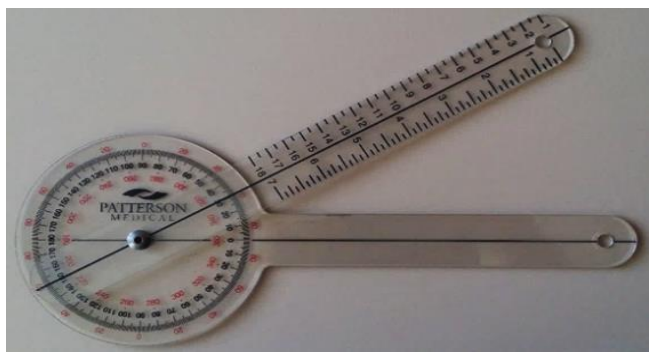

Fig. 1. Universal goniometer

- Gravity-dependent goniometer (inclinometer), BMI, Germany (Fig. 2). The scale of the inclinometer is graduated from 0 to $90^{\circ}$, the circular dial is divided into 4 sectors, the measuring capabilities are from 0 to $90^{\circ}$ and from 90 to $0^{\circ}$ clockwise and counterclockwise. The pointer is fixed in the center of the dial and is gravitationally balanced bilaterally.

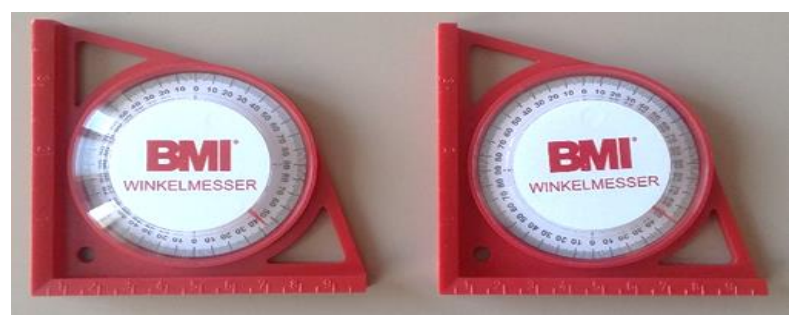

Fig. 2. Gravity-dependent goniometer

Six main directions of neck movement were investigated:

- Cervical flexion - the movement of the head in the sagittal plane around the medio-lateral axis is examined. The test position of the patient is seated in a chair with a well-fixed back of the backrest. The head position is $0^{\circ}$ lateral flexion and $0^{\circ}$ rotation. Stabilization of the patient is done by fixing the shoulder and back of the patient by the assistant. Test movement - the patient performs an active movement until the beard touches the chest. The reference upper limit for active mobility is $50^{\circ}$ according to the American Medical Association [16].

- Cervical extension - the movement of the head in the sagittal plane around the medial-lateral axis is examined. The test position of the patient is seated in a chair with a well-fixed back of the backrest. The 
head position is $0^{\circ}$ lateral flexion and $0^{\circ}$ rotation. Stabilization of the patient is done by fixing the shoulder and back of the patient by the assistant. Test movement - the patient actively moves the head back to the maximum end position. The reference upper limit for active mobility is $60^{\circ}$ according to the American Medical Association [16].

- Cervical lateral flexion - the movement of the head in the frontal plane around the anteriorposterior axis is investigated. Test position - the patient is seated and the position of the cervical spine is $0^{\circ}$ flexion, extension or rotation. The stabilization of the patient is done by fixing the shoulders and chest of the assistant. Test movement - the patient actively tilts the head alternately to the right and left shoulders, without rotating the head. The reference upper limit for lateral flexion is $45^{\circ}$ according to the American Medical Association [16].

- Cervical rotation - the movement of the head in the transversal plane around the axial axis of the body is investigated. Test Position - The patient is seated in a chair with the thoracic and lumbar spine tightly resting against the backrest. The cervical spine is in position $0^{\circ}$ with respect to flexion, extension and lateral flexion. The stabilization of the patient is performed to the back of the chair by assisting in order to prevent rotational movement in the lumbar department. Test Movement - The patient performs an active movement of head rotation consecutively to the far right and far left positions. The cervical rotation reference upper limit is $80^{\circ}$ according to the American Medical Association [16].

\section{Results.}

Sixty eight patients with neck dissections were examined. The cohort is represented by 25 women and 43 men, with a mean age (SD) of 61.18 years (13.66\%). Radical neck dissection (RND) was performed in 31 patients (46\%), suprachomichioid neck dissection (SOHND) was performed in 30 patients and selective neck dissection (SND) was performed in 7 patients (10\%).

Evaluation of the cervical spine: The values of active mobility corresponding to the existing reference limits, were registered preoperatively: flexion - 500, extension - 600, lateral flexion - 450, lateral rotation - 800.

Significant deviations from the reference values were recorded postoperatively at month 3: flexion $\mathrm{X}=27.40, \mathrm{SD}=7.91, \mathrm{CI}_{95 \%}=[25.48,29.31]$; extension $-\mathrm{X}=36.99, \mathrm{SD}=8.05, \mathrm{CI}_{95 \%}=[35.03,38.94]$; lateral flexion $-\mathrm{X}=27.65, \mathrm{SD}=7.78, \mathrm{CI}_{95 \%}=[25.76,29.53]$; lateral rotation $-\mathrm{X}=53.97, \mathrm{SD}=15.47, \mathrm{CI}_{95 \%}=[50.22$, 57.72]. A statistically significant difference was observed postoperatively at the 3rd month in all directions of movement studied in the three groups of operative interventions (neck dissection): flexion $\mathrm{F}(2,65)=643.566, \mathrm{p}<0.001$; екстензия $-\mathrm{F}(2,65)=445.045$, $\mathrm{p}<0.001$; lateral flexion $-\mathrm{F}(2,65)=314.077$, $\mathrm{p}<0.001$; rotation $-\mathrm{F}(2,65)=121.980, \mathrm{p}<0.001$.

A Tukey posthoc test was used to show that the arithmetic mean of the RND group for all motor directions flexion $(X=19.35)$, extension $(X=28.87)$, lateral flexion $(X=19.97)$ and rotation $(X=39.13)$ are statistically significantly different from the other two groups - SOHND and SND. No statistically significant difference was found between the arithmetic mean values for the rotation of the groups in SOHND and SND, $\mathrm{p}=0.207$ (фиг. 3, 4, 5, 6).

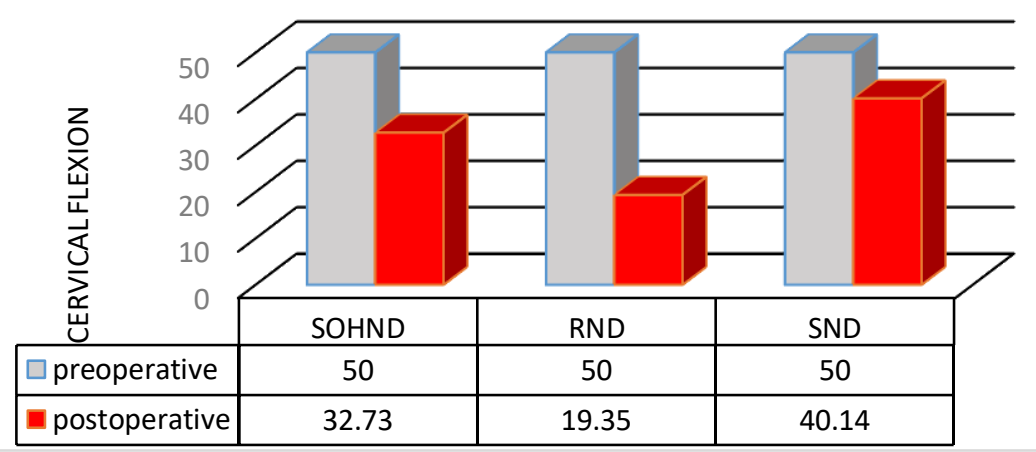

Fig. 3. Assessment of musculoskeletal function - AROM - flexion, preoperatively and postoperatively, in neck dissections 


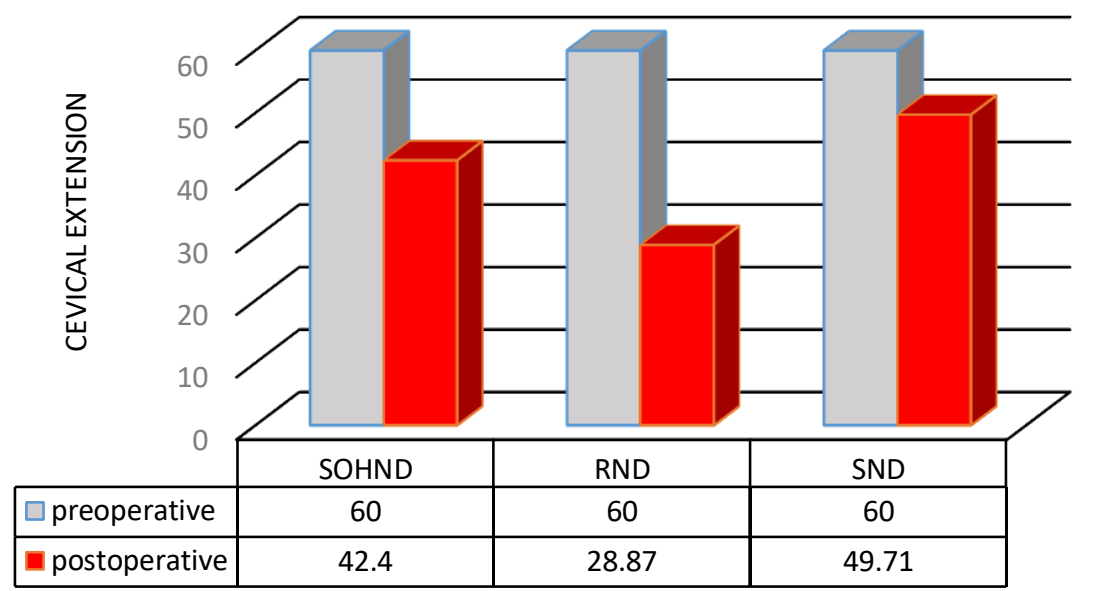

Fig. 4. Evaluation of the musculoskeletal system - AROM - extension, preoperatively and postoperatively, in neck dissections

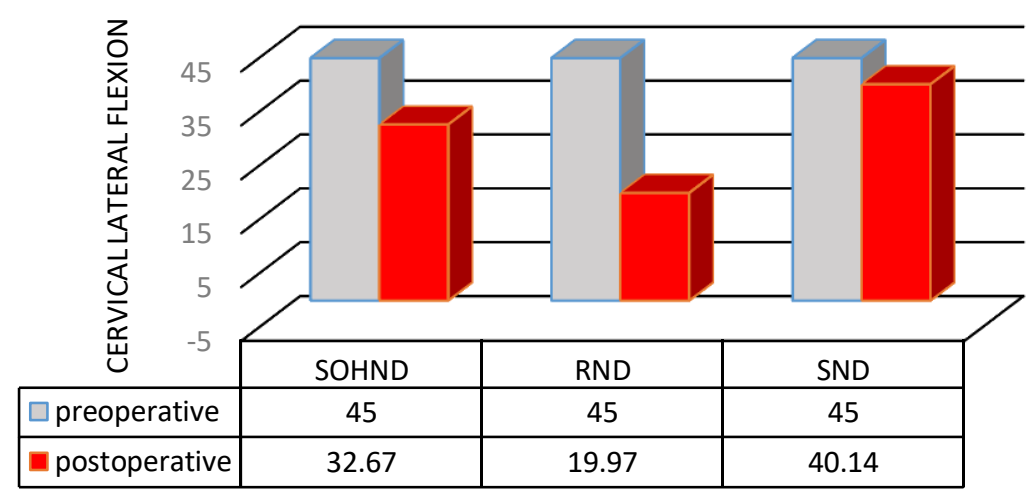

Fig. 5. Evaluation of the musculoskeletal system - AROM - lateral flexion, preoperatively and postoperatively, in neck dissections

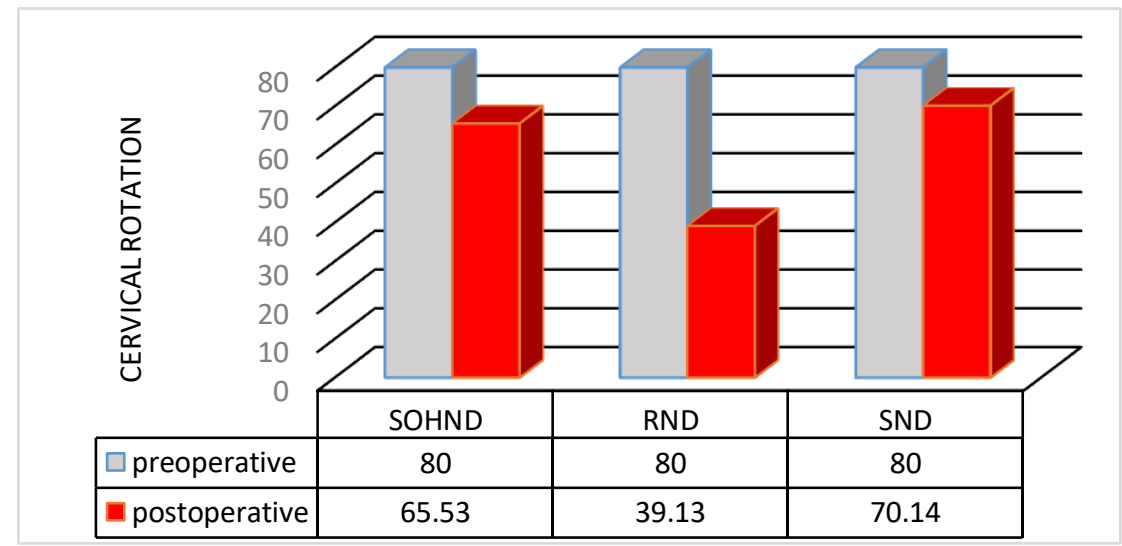

Fig. 6. Evaluation of the musculoskeletal system - AROM - rotation, preoperatively and postoperatively, in neck dissections

\section{Discussion.}

The deviations we found in the musculoskeletal system after surgical interventions of the neck - neck dissections, correspond with those established by other researchers [17].

C.P. van Wilgen et al. found that the range of motion of the cervical region was significantly reduced, with lateral flexion and ipsilateral involvement being significantly affected [15]. Ewing and Martin discuss the role of cicatricial change as a factor limiting cervical mobility [17]. Intensity transection of $\mathrm{n}$. accessorius and myotomy of $\mathrm{m}$. sternocleidomasttoideus cause marked musculoskeletal dysfunction [18, 17]. In a 
detailed study of the six directions of movement of the cervical spine, we find a significant difference between the degrees of motion in the different neck dissections.

In flexion and extension, the most significant limitation was found in RND, with significantly less movement disturbance in SOHND. Due to sacrificial behavior to a larger number of structures, RNDs have the highest postoperative morbidity: a neurotomy of $\mathrm{n}$. accessorius is performed, as well as a myotomy of MSCM. The unilateral removal of these structures is accompanied by asymmetry in the degree of mobility, the deflection of the head being ipsilateral to the operated side. Limiting motor activity in SOHND is associated with the development of postoperative cicatricial changes and the formation of coarse and dense features [17]. As a limitation in the movements flexion and extension at SOHND at the 3rd postoperative month are relatively significant, but due to their transitivity in time the movement of the neck at this operative volume is restored.

Lateral flexion of the head is significantly restricted to the side of surgery. This is related to neuronal and muscular damage in the RND. There is a limitation in the active degree of movement associated with the development of circulatory changes in the tissues. Head rotation is affected in the three variants of cervical dissection. In contrast to lateral flexion, the movement in the contralateral direction of surgery is impaired in rotation. This is related to the functional anatomy of the structures removed during surgery, with MSCM sacrifice being central. For this reason, we do not find a difference in head rotation between operated patients with SOHND and SND, where the function and continuity of this muscle are preserved.

\section{Conclusion.}

Assessment of the cervical spine shows that all directions of movement are affected, with the most significantly affecting of the lateral flexion in all groups of operated patients undergone neck dissection. The most pronounced cervical motility morbidity (active limitation and hypomobility) was observed in surgery in volume RND. In flexion and extension, identical changes were observed. Significant difference in restriction of neck movement was found in head rotation for patients with RND surgery.

\section{References}

[1] Ferlito, Alfio et al. Proposal for a Rational Classification of Neck Dissections. Wiley Periodicals, Inc. Head \& Neck, Version of Record online: 2010, DOI 10.1002/hed 21614; Head \& Neck, Editorial, 2011, 33, 3, 445-450

[2] Kesting, Marco. Oral Cancer Surgery: A Visual Guide. Georg Thieme Verlag KG, 2015, 132 pp. ISBN 978-3-13-199401-1, ISBN 978-3-13-199411-0 (e-book)

[3] Crile, G. W. On the surgical treatment of cancer of the head and neck. With a summary of one hundred and twenty-one operations performed upon one hundred and five patients. Trans South Surg Gynecol Assoc., 1905, 18, 108-127

[4] Gavilán Javier, Jesús Herranz, Lawrence W. DeSanto, César Gavilán. Functional and Selective Neck Dissection. Thieme, 2002, 179 pp. TMP ISBN 1-58890-016-9, GTV ISBN 3131246316

[5] Suárez, O. The problem of metastasis lymphatic cancer of the larynx and hypopharynx. Rev Otorrinolaringol., 1963, 23, 83-99. [Article in Spanish]

[6] Aleksiev E, M Nikolova, M Kondeva, L Videnova, P Stanimirov. Comorbidity and functional consequences after operative treatment of malignancies in head and neck area: Part I: comorbidity and sensory function, pain, speech and swallowing. - Medical Review, LIII, 2017, №6, 41-46

[7] Aleksiev E, M Nikolova, M Kondeva, L Videnova, P Stanimirov. Comorbidity and functional consequences after operative treatment of malignancies in head and neck area Part II: comorbidity, musculo-skeletal function and mental status. - Medical Review, LIV, 2018, №1, 34 - 40

[8] Aleksiev, E. Assessment of functional consequences after operative treatment of malignancy in maxillofacial and neck area. Dissertation thesis, Sofia, 2018. (In Bulgarian)

[9] Aleksiev, E. Functional consequences in surgical treatment in patients with maxillofacial and neck malignancy. - Medical Review, LIII, 2017, №4, 13-23

[10] Aleksiev E., L. Videnova, M. Petkova, M. Zekov, P. Stanimirov. Pain and sensory dysfunction in neck dissection due to neck metastatic diseases in malignant tumors at maxillofacial region. Modern Dentistry, XLVIII, 2017, №1-2, 10-19 
[11] Prim, M. P., J. I. de Diego, J. M. Verdaguer, N. Sastre, I. Rabanal. Neurological complications following functional neck dissection. Eur Arch Otorhinolaryngol., 2006, 263, 5, 473-476

[12] Tsekov M., M Mireva, K Guirov, S Marangozov, E. Aleksiev, Ch Videnova. Postsurgical rehabilitation of patients with superficial thrombophlebitis. Zdrave I nauka, VIII, 2018, 4, 35-38 (In Bulgaarian)

[13] Tsekov, M., K. Guirov, S. Marangozov, M. Mireva, E. Aleksiev, I. Lozev. Indications for emergency surgical intervention on superficial thrombophlebiti's patients. - Military medicine, 70, 2018, 2, 62-65

[14] Tsekov, M., K. Guirov, S. Marangozov, M. Mireva, E. Aleksiev, I. Lozev. Superficial thrombophlebitis - a social aspect of the disease. - Military medicine, 70, 2018, 4, 41-43.

[15] Van Wilgen, C. P., P. U. Dijkstra, B. F. van der Laan, J. T. Plukker, J. L. Roodenburg. Morbidity of the neck after head and neck cancer therapy. Head \& Neck, 2004, 26, 9, 785-791

[16] Norkin, Cynthia C., D. Joyce White. Measurement of Joint Motion. A Guide to Goniometry (4 ed.). F. A. Davis Company, 2009, USA, 468 pp. ISBN: 978-0-8036-2066-7, ISBN: 0-8036-2066-7

[17] EwIng, M. R. and H. Martin. Disability following ,radical neck dissection”. An assessment based on the postoperative evaluation of 100 patients. Cancer, 1952, 5, 873-883

[18] Kerawala, C. J. Complications of head and neck cancer surgery - Prevention and management. Oral Oncology, 2010, 46, 433-435 